\title{
WALLSY: The UWB and SmartMesh IP enabled Wireless Ad-hoc Low-power Localization SYstem
}

\author{
$1^{\text {st }}$ Sabin C. Gheorghiu \\ Dept. of Electronic and Electrical Engineering \\ University College London \\ London, United Kingdom \\ sabin.gheorghiu.16@ucl.ac.uk \\ $3^{\text {rd }}$ Rene H. Molnar \\ Dept. of Electronic and Electrical Engineering \\ University College London \\ London, United Kingdom \\ rene.molnar.16@ucl.ac.uk
}

\author{
$2^{\text {nd }}$ Kazmer Nagy-Betegh \\ Dept. of Electronic and Electrical Engineering \\ University College London \\ London, United Kingdom \\ kazmer.nagy-betegh.16@ucl.ac.uk \\ $4^{\text {th }}$ Ryan C. Grammenos \\ Dept. of Electronic and Electrical Engineering \\ University College London \\ London, United Kingdom \\ r.grammenos@ucl.ac.uk
}

\begin{abstract}
This paper follows the implementation of a proofof-concept localization system for GNSS-denied environments. WALLSY (Wireless Ad-hoc Low-power Localization SYstem) is a portable and modular Ultra Wide-Band (UWB) and Smart Mesh IP (SMIP) hybrid. WALLSY uses UWB two way ranging (TWR) to measure distances, which are then sent via the lowpower SMIP backbone network to a central hub for calculating coordinates of tracked objects. The system is highly flexible and requires no external infrastructure or prior knowledge of the installation site. It uses a completely nomadic topology and delivers high localization accuracy with all modules being battery powered. It achieves this by using a custom time-slotting protocol which maximizes deep-sleep mode for UWB. Battery life can be further improved by activating inertial measurement unit (IMU) filtering. Visualization of tracked objects and system reconfiguration can be executed on-the-fly and are both accessible to end users through a simple graphical user interface (GUI). Results demonstrate that WALLSY can achieve more than ten times longer battery lifetime compared to competing solutions (localizing every 30 seconds). It provides 3D coordinates with an average spatial error of $60.5 \mathrm{~cm}$ and an average standard deviation of $15 \mathrm{~cm}$. The system also provides support for up to 20 tags.
\end{abstract}

Keywords-WALLSY, UWB, TWR, Smart Mesh IP, ad-hoc, low-power, IMU, battery-powered, hybrid, sensor fusion, localization, GNSS-denied environments, nomad, modular, noninvasive, fully wireless

\section{INTRODUCTION}

\section{A. Background}

It is estimated that the global positioning system (GPS) has added $\$ 1.4$ trillion of value to the U.S. economy alone since its inception [1]. Indoor localization has been growing with a potential value equal or greater than that of GPS [2]. The choice of technology for indoors is not as straight forward as GPS is for outdoors. Due to various constraints and application requirements each technology used within indoor localization has different accuracy, range and power consumption characteristics [3].

Due to its superior accuracy, Ultra-Wideband (UWB) has gained significant ground as the preferred technology for indoor localization. UWB is also preferred for other GNSSdenied environments like short range outdoor applications which require sub-meter error range (generally tens of centimeters). The low interference compared to competing technologies as well as the ability to offer over a year of battery life for the tags (elements used to track objects/ people) have made it an ideal candidate [4] [5]. However, this is done with fixed and mains powered anchors (the reference points used within the localization system). Significant developments are still shaping how UWB impacts indoor positioning. Research [6] [7] on impulse-radio UWB (IRUWB) aims to increase communication robustness and enable very dense environments by using concurrent ranging especially in low data rate scenarios. Assembly line management and asset tracking in warehouses are an important driver of indoor localization with several solutions already in place or commercially available.

Other current UWB based systems that target improvements in tag battery life are again limited by the anchors. These need to be connected to mains power and in many cases require Ethernet cables [8] to communicate with each other. This hinders the ease of deployment and the overall system configurability. Such solutions are invasive and not modular making faults hard to troubleshoot. Furthermore, use cases are limited to indoor environments where the necessary cabling can be provided.

Battery powered anchors would enable the use of UWB localization systems in even more environments, particularly where setups only need to be temporary, examples including trade shows, festivals, sporting events, construction sites and refugee camps. Portable systems would enable significantly faster prototyping and testing of different deployment setups. There are already examples of UWB tracking solutions being used in sports events [9] but they still rely on semi-fixed cabling and the inherent high cost deters potential for other uses.

Localization systems which would satisfy the above needs would penetrate additional domains, for example, in drone swarm formation, where precise roaming network localization is paramount. Drones are poised to be used extensively in farming and field inspections [10] and are already used in the entertainment industry for light shows [11]. Portable, efficient, fully wireless and modular systems for localization would be key in formation flights.

The needed modularity and flexibility require such localization systems to be energy efficient and battery powered. One promising approach in the outdoors for improving precision even for reduced number of good localisation measurements is sensor fusion between GNSS and IMU [12]. Both precision and energy consumption on the tag stand to gain important improvements [13] [14] with sensor fusion between UWB and IMU. The obtained system was found suitable for navigation but was not tested in a realworld scenario and has not considered multiple objects in a 
formation. Processing overhead and power consumption were also not considered. It is worth mentioning that the obtained system is not GNSS specific and can be used with other sources for absolute distance measurement such as UWB.

The original contribution of WALLSY is the use of SMIP as a low-power backbone for UWB distance ranging. This has enabled a fully battery-powered system. The modularity and flexibility are complemented by high precision and low latency. This was achieved by designing a custom timeslotting protocol to operate and coordinate SMIP communications and UWB ranging measurements. Previous work using a single type of radio sacrifices either precision (for example, BLE) or the ability to be battery-powered (for example, UWB). Hybrid systems (for example, BLE with UWB) improve precision and battery lifetime at the expense of network reliability and system scaling.

This paper is organized as follows: Section I reviewed related work in the field; Section II describes the WALLSY system architecture; Section III presents measurements and discusses results while Section IV concludes this paper and makes recommendations for future work.

\section{B. Aims and Key Performance Indicators (KPIs)}

WALLSY aims to demonstrate the feasibility of a fully battery-powered indoor localization system with a custom low-power communication backbone and time-slotting protocol. The system has been designed to achieve a significant decrease in power consumption compared to available solutions [15] while delivering sub one-meter localization error, variable localization frequency and multitag support. The key requirements for the WALLSY system may be outlined as follows:

1. Modules must be battery powered and portable.

2. Latency ${ }^{\mathrm{a}}$ under 5 seconds, $\mathrm{Lag}^{\mathrm{b}}$ under 1 second.

3. Accuracy: Under 1 meter error $95 \%$ time, under 5 meters error at all times.

4. Fail-aware: Should localization fail, the system needs to identify this and explicitly notify the user.

5. Battery life: 1 week when localizing every 30 seconds.

6. Multi-tag: Support up to 20 tags.

7. Adjustable localization frequency: Support up to $1 \mathrm{~Hz}$.

8. Weight: Prototype should be under $300 \mathrm{~g}$ (approximately two times the weight of a smartphone ${ }^{\mathrm{c}}$ ).

9. Size: Fit in a $15 \mathrm{~cm}$ edge cube (the length of a smartphone ${ }^{\mathrm{c}}$ )

Latency $=$ time difference between the instant the tag is at location $\mathrm{X}$, and the instant the system learns location $\mathrm{X}$

$\mathrm{Lag}=$ time difference from the instant when the system learns of tag location to when the information is displayed on the user

The device taken as reference was an Apple iPhone 11 (weight: 188g, length: $15 \mathrm{~cm}$ ).

\section{SOlution OVERVIEW}

\section{A. System Outline and Operation}

The main challenge of creating a system with batterypowered UWB anchors is energy consumption. UWB measures distance by time of flight of the radio beam from one radio module to another. Provided the clocks on the devices are synchronized to nanosecond level, the distance can be determined to within a few centimeters. This is done by taking the difference between transmission and reception time and dividing it by the speed of light. The procedure is called "time difference of arrival" (TDOA). For the goals of WALLSY, TDOA is not a suitable option as the anchors need either wired synchronization or frequent wireless synchronization (every few hundred milliseconds) to accommodate clock drift within nanosecond-fine limits. Frequent transmissions are very energy inefficient. Using TDOA also requires that anchors precision clocks be kept in active state (high power) between synchronization events. This TDOA requirement prevents battery-powered anchor implementations.

Two way ranging (TWR) was the alternative employed. Previous synchronization is not needed and transmissions and receptions are very much reduced especially when ranging is not continuous. In between ranges the device can be kept in deep-sleep mode (consuming just 50nA). To achieve this, TWR basically combines synchronization and ranging in the same procedure. The reduction in power for anchors does translate into extra packet transmission being needed between each tag and anchor but the result is a much better distributed energy consumption of modules and, as such, is a good compromise.

Once distances between each tag-anchor pair are determined via UWB TWR they need to be aggregated on a central element which translates them into three dimensional (3D) coordinates. Although sending localization data via UWB is possible and actually employed by most solutions, it is very energy costly. Needed data rates are much lower than UWB bandwidth capability as such packet space is wasted. WALLSY makes use of Smart Mesh IP (SMIP) as a backbone system to connect four anchors and up to 20 tags. As shown by the cyan and dark blue colored numbers in Fig. 1, tags and anchors use UWB radio modules to subsequently determine the distance between each other.

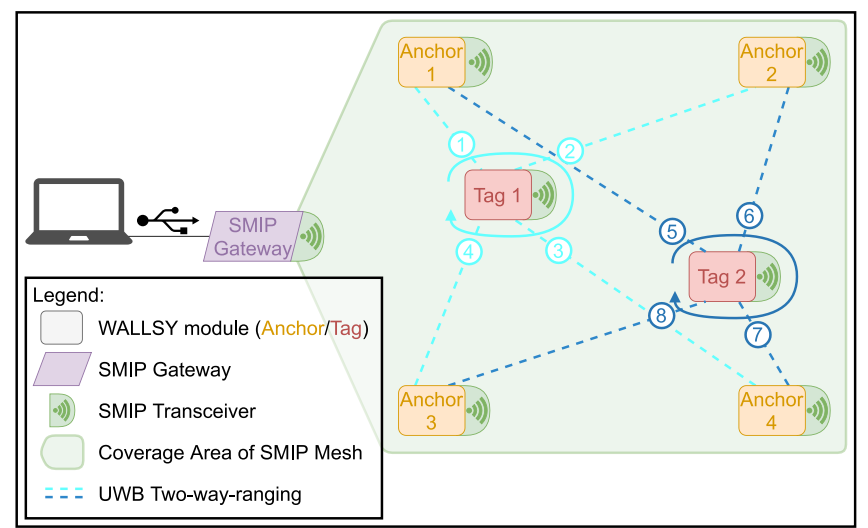

Fig. 1. WALLSY system outline

As presented in Fig. 2, WALLSY both tags and anchors have the same hardware architecture (SMIP radio, UWB radio, main microcontroller). SMIP transmission on anchors is used to send ranges back to the gateway for processing. Tag transmissions are only used for fault detection.

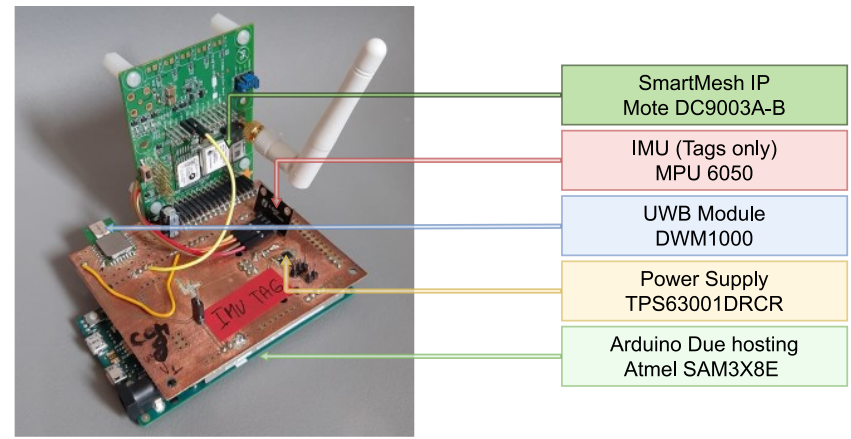

Fig. 2. WALLSY module as an early prototype. Labeled for differentiating. 
SMIP reception on all WALLSY modules is used to receive configuration information from the gateway. For the purpose of localisation all modules are hardware wise the same. This makes the elements of WALLSY interchangeable on-the-fly by reconfiguring which modules are anchors in the GUI. The system automatically deals with the change in topology and generates configuration for tags as well as updating the graphical user interface (GUI). This also provides very easy replacement and very low potential down time if a redundant number of anchors and tags is employed.

The central hub (SMIP gateway DC2274 and computer) uses the ranges combined with the $3 \mathrm{D}$ coordinates of the anchors to calculate the resulting 3D coordinates of each tag through a trilateration algorithm. Subsequently, with minimal lag, the position of the tags is displayed to the user on a twodimensional map on the GUI.

\section{B. Ultra-Wideband Module}

The DWM1000 UWB radio module from DecaWave was the one used in this work. It has sufficient precision $(10 \mathrm{~cm})$, can be integrated easily with external microcontrollers via the standard serial peripheral interface (SPI), has a small form factor (similar in size to a 20 euro cent coin) and a good balance between power draw and practical range. It achieves up to $250 \mathrm{~m}$ of coverage which is sufficient for the system purpose. The DWM1000 draws $160 \mathrm{~mA}$ and $140 \mathrm{~mA}$ peak current during reception and transmission mode, respectively. It only draws 50nA on average in deep-sleep mode [16]. The module consumes the most energy during its preamble hunt mode in reception mode so this needs to be reduced to a minimum.

\section{Inertial Measurement Unit (IMU)}

An MPU6050 module was added to aid power reduction. If IMU filtering is enabled, the accelerometer and gyroscope measurements are combined to extract an estimate of the movement of the WALLSY tag between ranging events. The algebraic formulas were partly based on research regarding second order derivatives for state estimation [17]. If the displacement value is lower than a set threshold (usually the mean error of UWB) then the unit does not need to range as it is quasi-stationary from a system point of view thus saving energy. The module will eventually need to range when the IMU accumulated drift becomes comparable to the threshold. This time has been empirically found to be around 20 minutes. Using IMU complements the UWB time slotting in contributing to energy reduction but can also be used to enhance localization precision.

\section{Smart Mesh IP Module}

The DC9003A-B SMIP radio module was developed by Dust Networks of Analog Devices [18]. It boasts an ad-hoc mesh network with $99.999 \%$ end-to-end reliability and ultralow power characteristics making it an ideal candidate for this application. The mesh network operates using a $10 \mu \mathrm{s}-\mathrm{level}$ synchronization, which can be accessed from each of the motes on the network. While this is certainly not precise enough for UWB TDOA, it is used to achieve synchronization of the needed time-slotting protocol.

\section{E. Time Slotting Protocol}

To reduce power consumption, the UWB module should only be turned on and in reception mode when a transmission is about to happen. This raises the need for a time slotting protocol. A visualization of the time-slotting period is presented in Fig. 3.

In addition to precise time information, the backbone network provides each component with the system configuration. This contains information about its role within the system (if it is a tag or an anchor) and its corresponding time slot to perform ranging. This results in the WALLSY modules turning on in sequence during allocated slots within the ranging superframe. For each slot, a different anchor is performing UWB TWR with the tag that is active in the corresponding frame. As anchors and tags can enter low power operation as often as possible, their UWB radio modules spend most time in deep-sleep mode, drastically reducing mean power requirements.

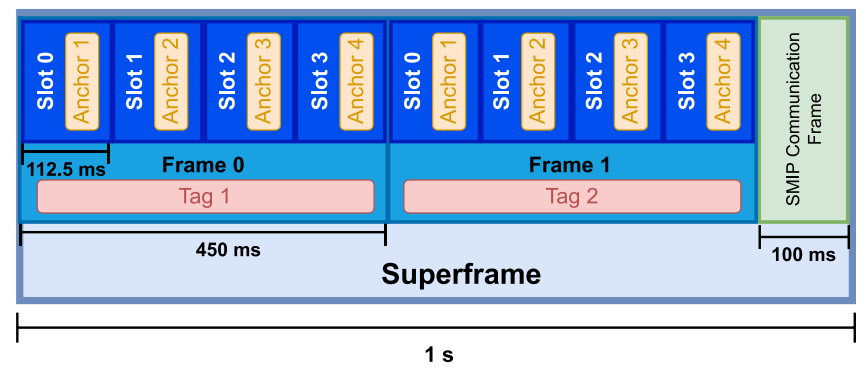

Fig. 3. Time slotting protocol example for a two-tag system (active anchor/tag shown next to slot/frame number)

The SMIP slot at the end of each frame is used to transmit the UWB ranging results accumulated on the anchors. It has a separately allocated slot for transmission after all ranges have been collected and compressed together in a single packet. This minimizes the chance of network overload.

\section{F. On-the-fly Configuration}

The information relating to tag activity, tag id, localization frequency, slot and frame and the total number of tags is shared from the gateway to all WALLSY modules in a single packet called the "config-file". This not only minimizes transmissions but also ensures configuration consistency. Every module knows when (slot and frame) and who (ID) they need to range with. This takes care of error handling and any invalidly configured modules can be picked up and signaled on the GUI.

Once the SMIP radio modules are online and have synced time with the master clock the config-file can be sent at any point. Any configuration changes (new tag joins, localization frequency changes) will be accompanied by sending the new config-file. This enables it to act as a trigger for forcerefreshing active tags in the next superframe. This is because all active tags range in the first superframe after the config-file is sent and this satisfies the latency KPI. SMIP packet size limitation meant that the information had to be assembled in the most efficient way possible. An example of the config-file is in Fig. 4.

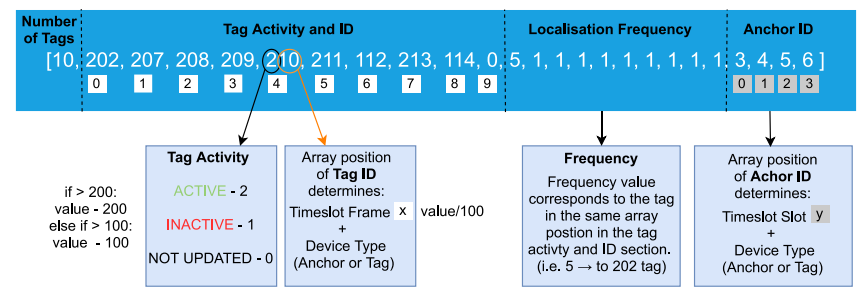

Fig. 4. Config-file example with 4 anchors and 10 tags 


\section{G. Vizualisation}

Based on the number of successful anchor ranges received at the central hub, the trilateration algorithm localizes with four anchors (giving a unique 3D location result) or three anchors (giving an XY coordinate and displaying a warning). Tags which are lost (have not ranged) are shown in a last position and with a last updated timestamp. Tags which are offline, or which skipped the current superframe (because their localization frequency was set to a reduced value) will have reported ranges replaced by a reserved code. Encoding information like this gives explicit awareness of tag states (IMU filtered/lost).

\section{H. Operation Logic of WALLSY Elements}

Algorithm 1 presents the logical operation of a system module. This firmware was written in $\mathrm{C} / \mathrm{C}++$. Algorithm 2 presents the code running on the central hub. The GUI and SMIP manager driver implementation were written in Python.
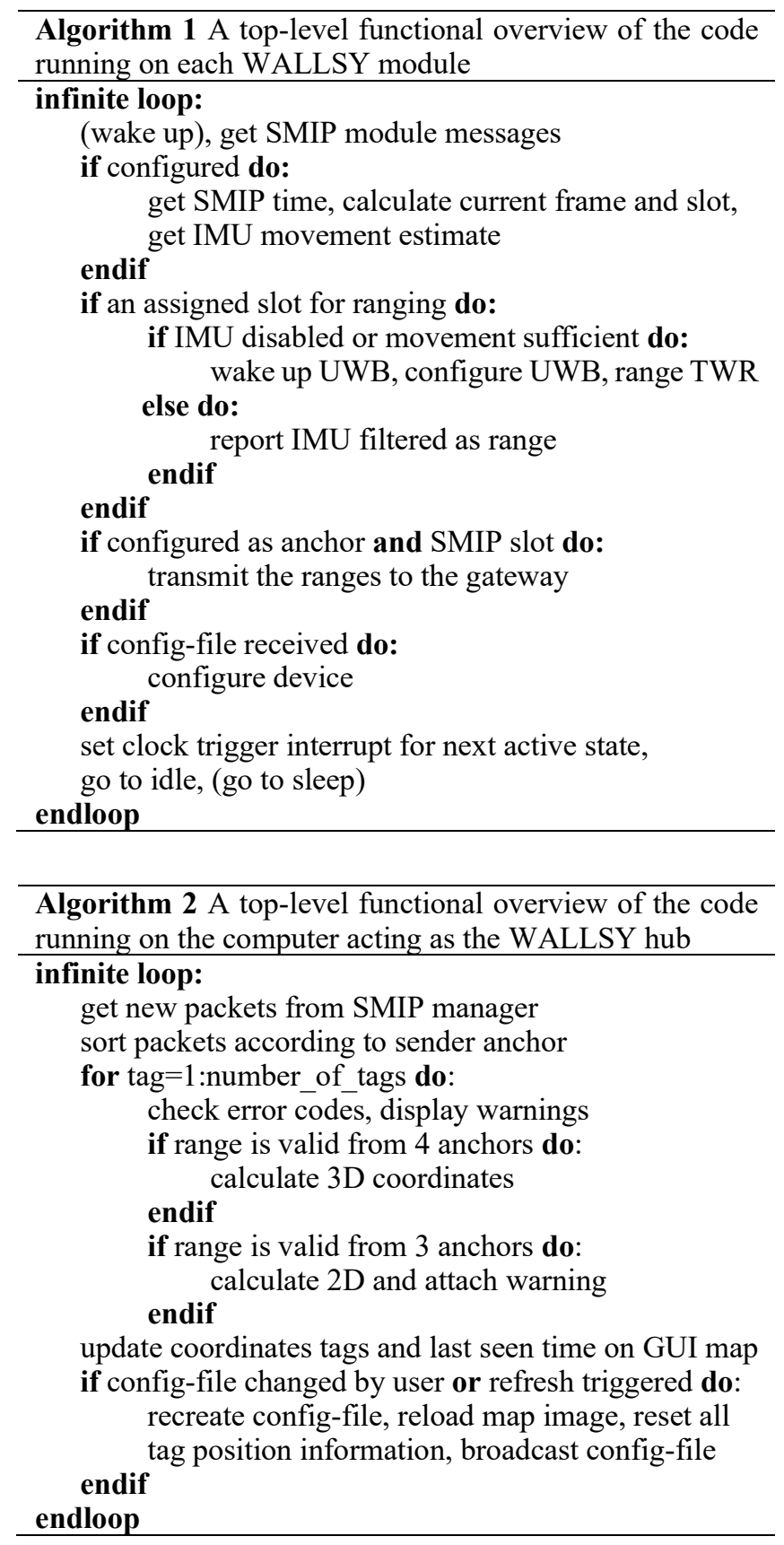

\section{MEASUREMENTS}

To determine the localization accuracy of WALLSY, real and reported coordinates of reference positions were measured and compared. Due to Covid-19 restrictions the only suitable test environment was a shared kitchen and dining area but results are expected to be similar in larger areas. The reference positions for anchors and tags in the test environment are shown in Fig. 5. The true coordinates were measured with a laser rangefinder.

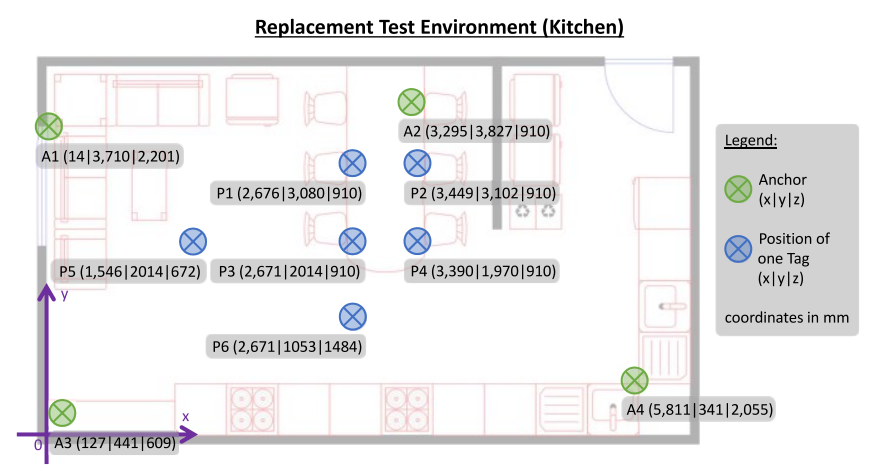

Fig. 5. Environment for testing localization accuracy

\section{A. 3D Localization Accuracy}

For each position, the system executed 1000 sequential localization actions and the corresponding outputs were recorded as $3 \mathrm{D}$ coordinates. The results for all six tested positions can be observed in Fig. 6 and Fig. 7 showing mean error, the standard deviation as well as maximum and minimum errors.
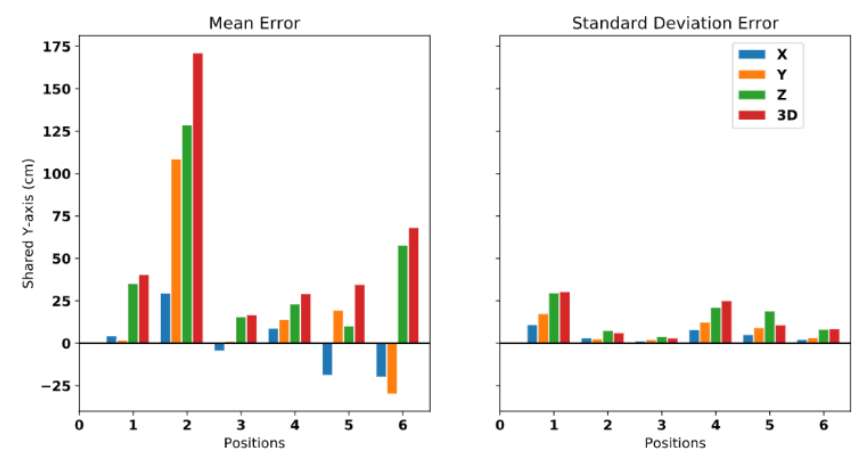

Fig. 6. Measured mean error and standard deviation of WALLSY
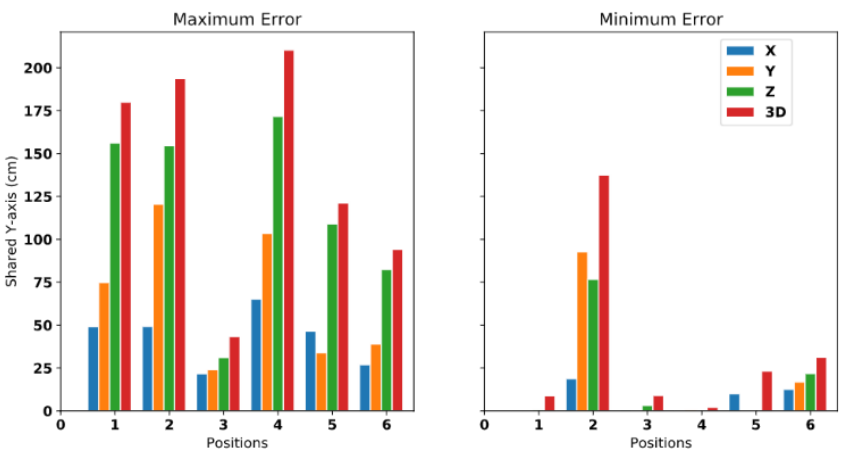

Fig. 7. Measured maximum and minium error of WALLSY

Considering the test environment in Fig. 5, the results are as expected. The largest error is present on the z-axis and this is due to a skewness on this axis (the difference in height between anchors is much lower than horizontal differences). It is also worth noting that position P2 presents the highest 
operation while providing a sufficient battery life, all in combination with a sub-one-meter localization error.

\section{System Limitations}

The current main limitations of WALLSY are power consumption and the number of supported tags. The following two paragraphs discuss how these two limitations can be overcome:

Power: Because the chosen Atmel SAM3X8E main microcontroller lacks support for sleep implementation, it is currently the main consumer of energy. This can be drastically improved with sleep implementation in idle phase (majority of time). The MSP430F5529LP microcontroller is a good replacement both in selection of ports and memory. Assuming the MSP430F5529LP runs at 1Mhz in LPM3 mode drawing $1.4 \mu \mathrm{A}[20]$ in idle phase, then the total energy draw for that phase could be considerably lowered. SMIP draws a mean current of $1191 \mu \mathrm{A}$ [21] and UWB draws 50nA in deep sleep [16]. As such, the new idle phase draw would be roughly $1.2 \mathrm{~mA}$ instead of the current $18.4 \mathrm{~mA}$ (reduction of more than 1 order of magnitude). This reduction would enable a battery life of almost half a year.

Number of Tags: Considering an independent system of four anchors, there are two main limitations regarding the number of supported tags:

- 43 tag limit - this is given by the maximum payload per SMIP packet as well as the maximum number of packets per second for the backbone network.

- 60 tag limit - The DWM1000 module needs minimum times for wake up and sleep transitions. With the implementation of pipelining the slots are used for ranging only. The ranging itself takes a minimum time of $3.7 \mathrm{~ms}$. Any further increase in tag number needs to consider overlapping and frequency channel slotting over the existing time slotting (assuming that the highest localization frequency is once per second and thus the superframe length cannot be longer than 1s).

\section{CONCLUSIONS}

This paper described the implementation of WALLSY, a proof-of-concept design of a SMIP and UWB enabled hybrid, battery-powered, modular and ad-hoc localization system.

The main challenge developing code was to achieve stable and consistent messaging over SMIP within the strict lowlatency requirement of the system. WALLSY achieves under 1 second latency and lag which is significantly lower than our initial KPI. The UWB module interaction programming presented the challenge of integrating ranging and deep-sleep mode in the custom time slotting algorithm.

WALLSY shows an increase in battery life of more than one order of magnitude (to approximately 13.54 days) compared to the competing Pozyx system (1.08 days). In situations where the tracked objects are not moving significantly (with regard to anchors), the IMU filtering enables even longer battery life. There is substantial potential to further reduce power consumption by using a low-power microcontroller as well as removing the debug port output and the status LEDs.

Real-time modification of parameters of the system is achieved through a configuration file which is easily interacted with in a GUI on the screen of the central hub. This enables the user to further optimize power consumption and tracking by adapting the localization frequency and the IMU filtering on the spot to the needs of the application.

WALLSY's portable nature and fully wireless installation make it a good fit for a myriad of applications. Tracking at temporary events like trade shows or pop-up events demand such battery powered and fully wireless systems with high precision (average spatial error of $60.5 \mathrm{~cm}$ ). These events generally last less than two weeks and would significantly benefit from the independence of permanent infrastructure in terms of required set-up time and set-up costs.

Experimental results showed that WALLSY provides accurate coordinates for tags positioned in arbitrary locations within the area defined by the anchors. Further preliminary experiments have also demonstrated WALLSY's ability to position tags on-the-fly by showing their real-time location on a rendered map [22]. Future work will look at evaluating the performance of WALLSY in a dynamic scenario.

An example of such a scenario would include drone swarms, where fast and accurate localization would aid optimal flight formation in applications ranging from visual effects in shows to sky corridors or farming. The applications for this kind of system stretch far beyond just localization of objects in a fixed environment into nomad networks found in collaborative localization. Together with the high precision, this enables indoor mapping or real time navigation implementation for asset tracking in warehouses.

Finally, we will look at addressing system limitations and improve system stability. Apart from the methods discussed in Section III.C the simplest way to increase the supported tag limit is using a longer superframe at the expense of a higher lag or slower localization frequencies. This enables more ranging frames to be inserted as well as more SMIP packets to be sent per superframe. Power consumption and precision would not be affected by this change.

WALLSY offers a highly precise and versatile platform for localization while also recalling the KPI's set out in Section I.B. With portable packaging, the system can be operated for approximately two weeks while reporting localization data on a simple GUI. The unique characteristics make it not only a good fit for the previously mentioned range of applications but they also enable many new ones.

\section{ACKNOWLEDGMENT}

We thank UCL's Electronic and Electrical Engineering department for supplying the components and instruments required to realize WALLSY as well as Mohammad Jabeen for his efforts in supporting this work. Finally, we are grateful to the team who worked on the WALDO project (the ultrasound-based predecessor of WALLSY) which inspired this work.

\section{REFERENCES}

[1] Mary-Deirdre Coraggio, "Economic Benefits of the Global Positioning System to the U.S. Private Sector Study," National Instituete of Standards and Technology, 2019. [Online]. Available: https://www.nist.gov/news-events/news/2019/10/economic-benefitsglobal-positioning-system-us-private-sector-study. [Accessed: 30Apr-2021].

[2] Deloitte, "2017 Technology, Media and Telecommunications Predictions - Middle East edition.” Deloitte Prediction Report, 2017.

[3] F. Zafari, A. Gkelias, and K. K. Leung, "A Survey of Indoor 
Localization Systems and Technologies," IEEE Commun. Surv. Tutorials, vol. 21, no. 3, pp. 2568-2599, 2019.

[4] Sewio, "UWB Tags for Indoor Location Tracking," 2021. [Online]. Available: https://www.sewio.net/uwb-tags/. [Accessed: 30-Apr2021].

[5] A. R. J. Ruiz and F. S. Granja, "Comparing Ubisense, BeSpoon, and DecaWave UWB Location Systems: Indoor Performance Analysis," IEEE Trans. Instrum. Meas., vol. 66, no. 8, pp. 2106-2117, 2017.

[6] P. Corbalán and G. Pietro Picco, "Ultra-wideband Concurrent Ranging," arXiv, vol. 1, no. 1, 2020.

[7] J. Colli-Vignarelli, J. Vernez, R. Merz, C. Dehollain, S. Robert, and J. Y. Le Boudec, "Concurrent transmissions in IR-UWB networks: An experimental validation," Proc. - 2009 IEEE Int. Conf. UltraWideband, ICUWB 2009, no. October, pp. 337-342, 2009.

[8] Pozyx, "Pozyx Enterprise System." [Online]. Available: https://pozyx.io/products-and-services/enterprise. [Accessed: 29Apr-2021].

[9] "Sportable sports tracking equipment." [Online]. Available: https://www.sportable.com/. [Accessed: 30-Apr-2021].

[10] W. Wang, N. Okati, I. Tanash, T. Riihonen, and E. S. Lohan, "Location-based beamforming architecture for efficient farming applications with drones," 2019 Int. Conf. Localization GNSS, ICLGNSS 2019 - Proc., 2019.

[11] "2021 London O2 drone light show," 2021. [Online]. Available: https://www.london.gov.uk/press-releases/mayoral/fireworkslighting-and-drones-welcome-2021. [Accessed: 30-Apr-2021].

[12] D. Imparato, J. Floch, E. Simona, M. Růžička, and P. Casek, “A Study on Robust Navigation by Means of GNSS, Network LTE and INS Integration," in International Conference on Localization and GNSS, 2020 .
[13] R. Moradi and M. Hutchinson, "Hybridized GNSS and IMU Positioning for Train Infrastructure Asset Health Status Monitoring within the SIA-Project," in International Conference on Localization and GNSS, 2020.

[14] M. Gunia, Y. Lu, N. Joram, and F. Ellinger, "On the Precision of Common Individual or Hybrid Positioning Systems," 2019 Int. Conf. Localization GNSS, ICL-GNSS 2019 - Proc., no. 3, 2019.

[15] Pozyx, "Pozyx Creator System." [Online]. Available: https://pozyx.io/products-and-services/creator-rtls/. [Accessed: 29Apr-2021].

[16] Decawave Ltd., "DW1000 Datasheet," pp. 11-13, 2015.

[17] R. Delpoux, H. Sira-Ramírez, and T. Floquet, "Acceleration feedback via an algebraic state estimation method," Proc. IEEE Conf. Decis. Control, no. December, pp. 5704-5709, 2013.

[18] T. Watteyne, L. Doherty, J. Simon, and K. Pister, "Technical overview of smartmesh IP," Proc. - 7th Int. Conf. Innov. Mob. Internet Serv. Ubiquitous Comput. IMIS 2013, pp. 547-551, 2013.

[19] L. Doherty, K. S. J. Pister, and L. El Ghaoui, "Convex position estimation in wireless sensor networks," Proc. - IEEE INFOCOM, vol. 3, pp. 1655-1663, 2001.

[20] Texas Instruments, "MSP430F552x , MSP430F551x Mixed-Signal Microcontrollers Datasheet.” 2020.

[21] I. Analog Devices, "Smart Mesh IP power estimator," 2016. [Online]. Available: https://www.analog.com/en/technical-articles/smartmeshpower-and-performance-estimator.html. [Accessed: 10-Feb-2020].

[22] S. Gheorghiu, K. Nagy-Betegh, R. Molnar, and M. Jabeen, "DEMO WALLSY: The UWB and SmartMesh IP enabled Wireless Ad-hoc Low-power Localization SYstem," 2020. [Online]. Available: https://youtu.be/QrP-d1dmXbI. [Accessed: 02-May-2021]. 